\title{
0564 FROM IDB TO INTEGRIS A NEW APPROACH TO HOSPITAL BASED INJURY SURVEILLANCE IT THE EU
}

G Furian*, R Bauer, B Laursen, R A Lyons, E van Beeck Correspondence: Austrian Road Safety Board (KfV), Department for Research and Knowledge Management, Schleiergasse 18 A-1100 Wien Austria 1100, Austria

\subsection{6/ip.2010.029215.564}

The establishment of specially designed injury surveillance systems is widely advocated as a prerequisite for the development and evaluation of injury prevention strategies. By international experience the EU Data Base (IDB) is considered the most appropriate data system to that avail in Europe. The IDB focus is to collect external cause information for all sectors of injury prevention. The IDB is based on a sample of hospitals in currently $13 \mathrm{EU}$ member states; the central online database is hosted by DG Sanco. The fact, however, that the IDB was designed as a stand-alone data system, proved to be an obstacle for its wider implementation in Europe. The FP7 project INTEGRIS (Improved Methodology for data collection on accidents and disabilities), 2008 to 2011, is a feasibility study for the improvement of the IDB methodology and cost efficiency through an integration of the IDB with the routine Hospital Discharge Registers (HDR) at the hospital level. The presentation shows the INTEGRIS data model, the operationalisation of the ICD-based indicators for disabilities, and the INTEGRIS hospital interface and transmission protocol for the central database application. Also first results of the evaluation of the INTEGRIS pilot will be included in view of the objectives of INTEGRIS, to provide population based indicators for the incidence of various external causes of injury morbidity, the incidence of long term and chronic disabilities from accidents, and the compliance with the quality criteria of the European Statistical System (Eurostat) and the EU Health Indicators (DG Sanco). 